\title{
Sleep deprivation increases oleoylethanolamide in human cerebrospinal fluid
}

\author{
Dagmar Koethe · Daniela Schreiber · Andrea Giuffrida $\cdot$ Christian Mauss · \\ Johannes Faulhaber · Bernd Heydenreich • Martin Hellmich · \\ Rudolf Graf · Joachim Klosterkötter · Daniele Piomelli · F. Markus Leweke
}

Received: 2 July 2008/ Accepted: 30 November 2008/Published online: 10 January 2009

(C) Springer-Verlag 2008

\begin{abstract}
This study investigated the role of two fatty acid ethanolamides, the endogenous cannabinoid anandamide and its structural analog oleoylethanolamide in sleep deprivation of human volunteers. Serum and cerebrospinal fluid (CSF) samples were obtained from 20 healthy volunteers before and after a night of sleep deprivation with an interval of about 12 months. We found increased levels of
\end{abstract}

D. Koethe, D. Schreiber and A. Giuffrida contributed equally to this study.

D. Koethe $\cdot$ D. Schreiber $\cdot$ C. Mauss - J. Faulhaber .

B. Heydenreich · J. Klosterkötter · F. M. Leweke

Department of Psychiatry and Psychotherapy,

University of Cologne, 50924 Cologne, Germany

D. Schreiber · D. Piomelli

Department of Pharmacology, University of California,

Irvine, CA 9269, USA

D. Schreiber · D. Piomelli

Department of Biological Chemistry, University of California, Irvine, CA 9269, USA

A. Giuffrida

Department of Pharmacology, University of Texas Health

Science Center, San Antonio, TX 78229, USA

\section{Hellmich}

Institute of Medical Statistics, Informatics, and Epidemiology,

University of Cologne, 50924 Cologne, Germany

R. Graf

Max Planck-Institute of Neurological Research,

Cologne, Germany

F. M. Leweke $(\bowtie)$

Central Institute of Mental Health Mannheim,

University of Heidelberg, J5, 68159 Mannheim, Germany

e-mail: leweke@cimh.de oleoylethanolamide in CSF ( $P=0.011)$ but not in serum $(P=0.068)$ after $24 \mathrm{~h}$ of sleep deprivation. Oleoylethanolamide is an endogenous lipid messenger that is released after neural injury and activates peroxisome proliferatoractivated receptor- $\alpha$ (PPAR- $\alpha$ ) with nanomolar potency. Exogenous PPAR- $\alpha$ agonists, such as hypolipidemic fibrates and oleoylethanolamide, exert both neuroprotective and neurotrophic effects. Thus, our results suggest that oleoylethanolamide release may represent an endogenous neuroprotective signal during sleep deprivation.

Keywords Oleoylethanolamide - Endocannabinoids . PPAR- $\alpha \cdot$ Sleep deprivation $\cdot$ Neuroprotection .

Oxidative stress

\section{Introduction}

Oleoylethanolamide is a fatty acid ethanolamide (FAE) and a natural analog of the endogenous cannabinoid anandamide. There is no detailed research on the role of endocannabinoids in sleep in humans. Anandamide is known to engross slow-wave sleep by increasing adenosin levels in the forebrain of rodents (Murillo-Rodriguez et al. 2003). This is blocked by the cannabinoid $\mathrm{CB}_{1}$-receptor antagonist rimonabant. Oleamide is an endogenous sleepinducing lipid with putative cannabinomimetic properties (Lees and Dougalis 2004). Murillo-Rodriguez et al. (2007) supposed oleoylethanolamide, which-unlike oleamideactivates the nuclear peroxisome proliferator-activated receptor- $\alpha(\mathrm{PPAR}-\alpha)$ to increase alertness and to participate in the regulation of waking.

Up to now, elevated levels of oleoylethanolamide and anandamide were found in human microdialysates within the first day of ischemia as well as following neural injury 
or other stressors associated with necrosis. Furthermore, massive increases in FAEs and their precursor phospholipids have been found during the acute phase of stroke in the adult rat brain (for review, see Pacher et al. 2006). Thereby it was suggested that increases of brain FAE levels serve a neuroprotective function mediated by $\mathrm{CB}_{1}$-receptors. Oleoylethanolamide does not bind to cannabinoid CBreceptors but to PPAR- $\alpha$, thereby activating a different neuroprotective mechanism.

Sleep deprivation has been hypothesized to represent an oxidative challenge for the brain and that sleep may have a protective role against oxidative damage. While Gopalakrishnan et al. (2004) found no change in antioxidant enzymatic activities or increased oxidant production in the brain or in peripheral tissues after prolonged sleep deprivation, other studies suppose that sleep deprivation may result in a condition of oxidative stress including a reduction in glutathione levels in whole brains of rats (D'Almeida et al. 1998). Ramanathan et al. (2002) showed that prolonged sleep deprivation results in significant decreases in the activity of superperoxide-dismutase in rat hippocampus and brainstem. This effect may be due to the degradation of antioxidative enzymes after prolonged activation during wakefulness, which suggests an alteration in the metabolism resulting in oxidative stress. Even if sleep deprivation might not show extensive effects comparable to cerebral injury or tissue necrosis, FAEs have been shown previously to be elevated during situations of cellular stress and oxidative stress factors are elevated following sleep deprivation (Pacher et al. 2006). Therefore, in this study, we investigated whether the levels of the endogenous PPAR- $\alpha$ agonist oleoylethanolamide and the endocannabinoid anandamide were elevated in CSF and serum of healthy individuals after acute sleep deprivation.

\section{Methods}

The Ethics Committee of the Medical Faculty of the University of Cologne reviewed and approved the protocol of this study and the procedures for sample collection and analysis. All volunteers gave written informed consent twice at least 1 day prior to each lumbar puncture after extensive introduction into the procedures and goal of this study. The healthy subjects received an allowance for the participation in this trial. All investigations were conducted in accordance with the Declaration of Helsinki.

This study was integral to a larger project on endocannabinoid levels in CSF and serum of healthy volunteers and patients suffering psychiatric disorders. As part of that, volunteers were investigated to establish baseline levels of endocannabinoids in a healthy control population (Giuffrida et al. 2004). Twenty healthy volunteers (eight males and twelve females; median age 26 years, range 1949 years.) with no family history and no clinical indication for any relevant medical, psychiatric or neurological disturbances were included in our study to investigate effects of sleep withdrawal on endocannabinoid levels. Necessary criteria for inclusion were absence of cannabis use within the last year and lifetime cannabis use not exceeding five times. No positive urine drug screening for illicit drugs was accepted at time of the lumbar puncture (LP). Living circumstances (e.g. job status, social environment) for those volunteers selected did not change substantially during this period.

Subjects were lumbar-punctured twice during the course of our study using a nontraumatic LP procedure. The first LP was done under regular sleep condition. The second LP took place after $24 \mathrm{~h}$ of sleep deprivation about 1 year later to avoid seasonal influences and artificial alterations in cerebrospinal fluid (CSF) due to a previous LP in the near past. All subjects spent the nights before both LPs at home; sleep quality before the first lumbar puncture was assessed by the self-rating questionnaire for fatigue and for sleep and awakening quality (SSS). The night of sleep deprivation was spent in the habitual environment of the volunteers.

Alertness was monitored by wrist actigraphy, using the "Actiwatch2000," a piezoelectric transductor recording a maximum of 240 wrist motions per minute. Actiwatch was given to the volunteers $24 \mathrm{~h}$ before the second lumbar puncture. Volunteers showing a mosaic of inactivation at the actiwatch-scan of more than 5 min during the 24-h period were excluded from the experiment. This approach allowed us to continuously monitor the subjects during the night of sleep withdrawal (Benson et al. 2004).

Sleep habits in volunteers were assessed for 7 days before sleep deprivation using a sleep protocol to exclude interferences of the sleep rhythm. For sleep deprivation, volunteers were requested to stay awake for one night without consuming any coffee or alcohol during that night; intake of food was allowed. All LPs were done between 10:00 and 12:00 AM, and each time peripheral blood was collected. All samples revealed no pathognomonic cell counts, CSF/serum albumin ratios or oligoclonal bands. Virological and microbiological testing of the CSF was negative in all cases.

In all subjects, we measured CSF and serum levels of oleoylethanolamide along with anandamide. To quantify FAEs, CSF aliquots $(1 \mathrm{ml})$ and serum were spiked with 25 pmol of $\left[{ }^{2} \mathrm{H}_{4}\right]$-anandamide and $\left[{ }^{2} \mathrm{H}_{4}\right]$-oleoylethanolamide (internal standards) and prepared for further analysis as described previously (Schreiber et al. 2007). Fatty acid ethanolamides were purified and quantified by isotope dilution high performance liquid chromatography/mass 
spectrometry (HPLC/MS) using a HP 1100 Series HPLC/ MS system equipped with a octadecylsilica (ODS) Hypersil column $\left(100 \times 4.6 \mathrm{~mm}^{2}\right.$, i.d. $\left.5 \mu \mathrm{m}\right)$ (Agilent Technologies). MS analyses were performed with an electro-spray ion source as previously described (for details, see Giuffrida et al. 2004)

Statistical analyses were performed using SPSS and R software (library: exactRankTests). Because of apparent non-normality of empirical data distributions, location differences in (paired) measurements were assessed by Wilcoxon signed rank or rank sum test (exact).

\section{Results}

The measurements of oleoylethanolamide and anandamide levels in CSF of 20 healthy volunteers before and after $24 \mathrm{~h}$ sleep deprivation are illustrated in Fig. 1. Oleoylethanolamide levels in CSF (median 0.62, interquartile range 0.34-2.60 pmol/ml) increased significantly after $24 \mathrm{~h}$ of sleep deprivation $[7.44,1.18-20.14 \mathrm{pmol} / \mathrm{ml} ; P=0.011$ ( $P=0.044$, Bonferroni-adjusted)]. In serum, the increase from $7.70,6.13-15.00 \mathrm{pmol} / \mathrm{ml}$ to $12.34,6.80-21.21 \mathrm{pmol} /$ $\mathrm{ml}$ was borderline significant $(P=0.068)$. Anandamide levels were neither affected in CSF nor in serum ( $P=0.887$ and $P=0.229$, respectively). Notably, the oleoylethanolamide level increase in CSF was more pronounced in women $(19.52,0.68-23.39 \mathrm{pmol} / \mathrm{ml})$ than in men $(0.84,0.47-5.84 \mathrm{pmol} / \mathrm{ml}, P=0.211)$. There were no significant effects observed on age or gender on study variables. We found no difference between the self-reported

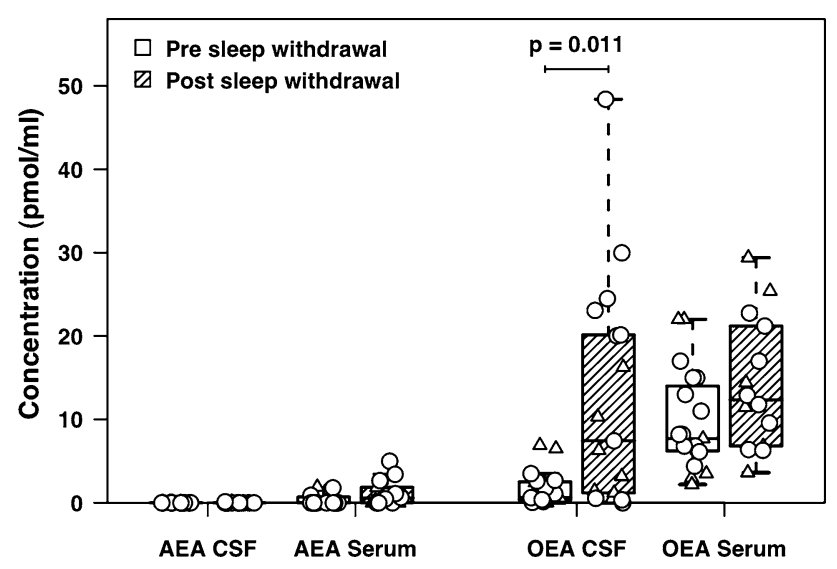

Fig. 1 Box-whiskers-plots (box shows 25th, 50th and 75th percentile of empirical distribution; whiskers extend to smallest and largest value excluding outliers) of acid ethanolamides, oleoylethanolamide and anandamide, in human cerebrospinal fluid (CSF) and in serum before and after a single night of controlled sleep withdrawal in healthy volunteers (circles: women, triangles: men). The $P$ value (two-sided) is from exact Wilcoxon signed rank test hours of sleep 7 days before first and second LP $(7.2 \pm 1.3 \mathrm{~h}$ vs. $7.5 \pm 1.4 \mathrm{~h})$. No mean metabolomic markers were changed between the first and second LP (as e.g. in clinical chemistry, sex hormones or BMI). Cortisol levels were measured as a stress marker in both conditions and revealed no change between first and second lumbar puncture $(156.2 \pm 70.2 \mathrm{nmol} / \mathrm{l}$ vs. $167.4 \pm 90.1 \mathrm{nmol} / \mathrm{l}$; $P=0.691)$. For serum glucose, no differences were found between regular and sleep withdrawal conditions $(87.40 \pm 14.3$ vs. $87.16 \pm 16.8 \mathrm{mg} / \mathrm{dl} ; P=0.71)$.

\section{Discussion}

Our results show a significant increase of oleoylethanolamide in human CSF after $24 \mathrm{~h}$ of sleep deprivation, whereas the levels of the endocannabinoid anandamide remain unaffected. Interestingly, there is no indication for a functional role of anandamide in sleep induction in humans as hypothesized previously (Mechoulam et al. 1997). In rats, Murillo-Rodriguez et al. (2006) showed that anandamide increases slow wave sleep, which can be blocked by administration of the cannabinoid $\mathrm{CB}_{1}$-receptor antagonist rimonabant, indicating that the endocannabinoid system is involved in sleep regulation (Murillo-Rodriguez et al. 2001).

Further, recent data in rats indicate diurnal variations of anandamide and oleoylethanolamide in CSF with maximum values for oleoylethanolamide during the late light phase, decreasing in the dark phase (Murillo-Rodriguez et al. 2006). This research was done in rats, which are awake in the lights-off period. Translating these results to the human sleep/wake cycle it may be hypothesized that the concentration of oleoylethanolamide in CSF (in contrast to brain tissue) may decrease during daytime and increase during sleep. Given these assumptions, the increase of oleoylethanolamide is not very likely the result of an accumulation, particularly with regard to the very limited extracellular life span of endocannabinoids (Piomelli 2003). As in our study the second lumbar puncture was done at the same time of the day as the first one; circadian rhythms should not confound our results. Oleoylethanolamide is renowned for modulation of feeding, body weight and lipid metabolism, as its levels decrease during food deprivation and increase upon feeding ( $\mathrm{Fu}$ et al. 2007). This should not be a confounder as well, as oleoylethanolamide plays its role as a local satiety hormone in the intestine (Lo Verme et al. 2005), and the feeding conditions were not changed before and after sleep-withdrawal in this study.

Like anandamide, oleoylethanolamide is synthesized by neurons and other cells in a stimulus-dependent manner and is rapidly eliminated by enzymatic hydrolysis 
(for review, see Pacher et al. 2006). Oleoylethanolamide binds with high affinity to the PPAR- $\alpha$, a nuclear receptor that is known to regulate several aspects of lipid metabolism and induces satiety by activating PPAR- $\alpha$ (Fu et al. 2003). PPAR $-\alpha$ is also involved in neuroprotection through activation of antioxidant and anti-inflammatory mechanisms. It has been shown that pretreatment with the synthetic PPAR- $\alpha$ agonist fenofibrate is neuroprotective against cerebral injury (e.g., Deplanque et al. 2003). Furthermore, treatment with PPAR- $\alpha$ agonists as well as monounsaturated and polyunsaturated fatty acids (PUFA) induces an increase in activity of major antioxidant enzymes in the brain (Deplanque et al. 2003).

The distribution of PPAR- $\alpha$ receptors in the human brain remains mainly conjectural. Moreno et al. (2004) have provided an overview on the distribution of PPARs in the adult rat central nervous system (CNS). They reported the highest densities of PPAR- $\alpha$ in the hippocampal dentate gyrus and the granular cell layer of the cerebellar cortex. Furthermore, they observed PPAR expression in previously unreported locations, such as the basal ganglia, the reticular formation, some thalamic, mesencephalic and cranial motor nuclei and the large motor neurons of the spinal cord (Moreno et al. 2004). This widespread distribution in the CNS also includes brain areas involved in sleep regulation such as the thalamus and the reticular formation.

It has been hypothesized that sleep may serve an antioxidant function by removing free radicals or oxygen reactive species produced during waking time and that restoration of antioxidant balance is a property of recovery sleep (Everson et al. 2005). However, these protective mechanisms, especially in the brain, are still poorly understood. Interestingly, several studies have found that sleep deprivation is a stressful condition, which is associated with the disruption of various physiological processes (McEwen 2006). Reduced glutathione levels have been found in different brain regions, such as thalamus and hypothalamus, as well as in different organs of sleepdeprived animals (D'Almeida et al. 1998; Everson et al. 2005). Additionally, markers of generalized cell injury accompanied these decreases (Everson et al. 2005) and decreased superoxide-dismutase activity has been shown in hippocampus and brainstem after prolonged sleep deprivation (Ramanathan et al. 2002). The activation of these components of the antioxidant defense system suggests that sleep deprivation is a stressful condition for the entire body and for the brain in particular.

Several studies have shown that PPAR- $\alpha$ activation enhances antioxidative enzyme activities (Deplanque et al. 2003). Activation of PPAR- $\alpha$ in vivo causes an upregulation of the mRNA and protein levels of a number of peroxisome- and nonperoxisome-associated enzymes and structural proteins, including the antioxidant enzymes catalase, superperoxide-dismutase and mediators of the glutathione pathway. In this context, pretreatment with fenofibrate reduces cerebral infarct volume in apolipoprotein E-deficient mice. The neuroprotective effect of fenofibrate is completely absent in PPAR- $\alpha$-deficient mice, suggesting that PPAR- $\alpha$ activation is involved as a protective mechanism against cerebral injury (Deplanque et al. 2003).

We failed to confirm the initially hypothesized increase of anandamide after sleep deprivation questioning its proposed role in sleep induction in humans (Mechoulam et al. 1997). This is in line with findings on cannabinoid $\mathrm{CB}_{1^{-}}$ receptor gene expression, which is suggested to be modulated by sleep. While sleep rebound significantly increased $\mathrm{CB}_{1}$-receptor protein and decreased respective mRNA, no effects were found following sleep deprivation (MartinezVargas et al. 2003). Interestingly, according to the product information, the $\mathrm{CB}_{1}$-receptor antagonist rimonabant induces sleep disturbances frequently (range 1-10\%) but also sedation (range $0.1-1 \%$ ) in clinical trials.

Several shortcomings might have influenced our somehow preliminary data; first, a randomized, balanced crossover design would have been the ideal design for the trial. Second, EEG recordings might have provided more detailed information on sleep quality instead of an actigraphy for control of sleep deprivation only.

\section{Conclusions}

Our results show that sleep deprivation significantly elevates the endogenous PPAR- $\alpha$ ligand oleoylethanolamide in human CSF. These findings may suggest the hypothesis that elevation of oleoylethanolamide may serve a potential neuroprotective role during sleep deprivation via activation of PPAR- $\alpha$. Despite its limits, this pilot trial addresses for the first time the role of anandamide and oleoylethanolamide in sleep regulation in humans. However, various questions remain unanswered: Which physiological stimuli initiate and terminate oleoylethanolamide biosynthesis in brain? Does oleoylethanolamide act on PPAR- $\alpha$ receptors in the brain as it does in the periphery? Does activation of PPAR- $\alpha$ elicit a neuroprotective response during sleep deprivation? These questions need to be addressed by further studies translationally targeting the role of oleoylethanolamide during sleep deprivation.

Acknowledgments We gratefully acknowledge the participation of Dr. Christoph W. Gerth and Dr. Miriam A. Neatby in the investigation of volunteers and we thank Dr. Regina Mangieri and Dr. Jesse LoVerme and Elisabeth New for reading the manuscript critically. This study was supported by the Stanley Medical Research Institute (01-315 to FML), the Koeln Fortune Program (108-2000 to FML), NARSAD (Staglin Family Vineyard Musical Fellowship to DP), the 
National Institute of Drug Abuse (DA12413 and DA12653 to DP), the National Institute of Neurological Disorders and Stroke (NS 050402 to AG), the National Institute of Diabetes and Digestive and Kidney Diseases (DK073955) and the German Federal Ministry of Education and Research (01KN0706 to DK).

\section{References}

Benson K, Friedman L, Noda A, Wicks D, Wakabayashi E, Yesavage J (2004) The measurement of sleep by actigraphy: direct comparison of 2 commercially available actigraphs in a nonclinical population. Sleep 27:986-989

D'Almeida V, Lobo LL, Hipolide DC, de Oliveira AC, Nobrega JN, Tufik S (1998) Sleep deprivation induces brain region-specific decreases in glutathione levels. Neuroreport 9:2853-2856

Deplanque D, Gele P, Petrault O, Six I, Furman C, Bouly M, Nion S, Dupuis B, Leys D, Fruchart JC, Cecchelli R, Staels B, Duriez P, Bordet R (2003) Peroxisome proliferator-activated receptor-alpha activation as a mechanism of preventive neuroprotection induced by chronic fenofibrate treatment. J Neurosci 23:6264-6271

Everson CA, Laatsch CD, Hogg N (2005) Antioxidant defense responses to sleep loss and sleep recovery. Am J Physiol Regul Integr Comp Physiol 288:R374-R383

Fu J, Gaetani S, Oveisi F, Lo Verme J, Serrano A, Rodriguez De Fonseca F, Rosengarth A, Luecke H, Di Giacomo B, Tarzia G, Piomelli D (2003) Oleylethanolamide regulates feeding and body weight through activation of the nuclear receptor PPARalpha. Nature 425:90-93

Fu J, Astarita G, Gaetani S, Kim J, Cravatt BF, Mackie K, Piomelli D (2007) Food intake regulates oleoylethanolamide formation and degradation in the proximal small intestine. $J$ Biol Chem 282:1518-1528

Giuffrida A, Leweke FM, Gerth CW, Schreiber D, Koethe D, Faulhaber J, Klosterkötter J, Piomelli D (2004) Cerebrospinal anandamide levels are elevated in acute schizophrenia and are inversely correlated with psychotic symptoms. Neuropsychopharmacology 29:2108-2114

Gopalakrishnan A, Ji LL, Cirelli C (2004) Sleep deprivation and cellular responses to oxidative stress. Sleep 27:27-35

Lees G, Dougalis A (2004) Differential effects of the sleep-inducing lipid oleamide and cannabinoids on the induction of long-term potentiation in the CA1 neurons of the rat hippocampus in vitro. Brain Res 997:1-14

Lo Verme J, Gaetani S, Fu J, Oveisi F, Burton K, Piomelli D (2005) Regulation of food intake by oleoylethanolamide. Cell Mol Life Sci 62:708-716
Martinez-Vargas M, Murillo-Rodriguez E, Gonzalez-Rivera R, Landa A, Mendez-Diaz M, Prospro-Garcia O, Navarro L (2003) Sleep modulates cannabinoid receptor 1 expression in the pons of rats. Neuroscience 117:197-201

McEwen BS (2006) Sleep deprivation as a neurobiologic and physiologic stressor: allostasis and allostatic load. Metabolism 55:S20-S23

Mechoulam R, Fride E, Hanus L, Sheskin T, Bisogno T, Di Marzo V, Bayewitch M, Vogel Z (1997) Anandamide may mediate sleep induction. Nature 389:25-26

Moreno S, Farioli-Vecchioli S, Ceru MP (2004) Immunolocalization of peroxisome proliferator-activated receptors and retinoid $x$ receptors in the adult rat CNS. Neuroscience 123:131-145

Murillo-Rodriguez E, Cabeza R, Mendez-Diaz M, Navarro L, Prospero-Garcia O (2001) Anandamide-induced sleep is blocked by SR141716A, a CB1 receptor antagonist and by U73122, a phospholipase C inhibitor. Neuroreport 12:2131-2136

Murillo-Rodriguez E, Blanco-Centurion C, Sanchez C, Piomelli D, Shiromani PJ (2003) Anandamide enhances extracellular levels of adenosine and induces sleep: an in vivo microdialysis study. Sleep 26:943-947

Murillo-Rodriguez E, Desarnaud F, Prospero-Garcia O (2006) Diurnal variation of arachidonoylethanolamine, palmitoylethanolamide and oleoylethanolamide in the brain of the rat. Life Sci 79:30-37

Murillo-Rodriguez E, Vazquez E, Millan-Aldaco D, Palomero-Rivero M, Drucker-Colin R (2007) Effects of the fatty acid amide hydrolase inhibitor URB597 on the sleep-wake cycle, c-Fos expression and dopamine levels of the rat. Eur $\mathrm{J}$ Pharmacol 562:82-91

Pacher P, Batkai S, Kunos G (2006) The endocannabinoid system as an emerging target of pharmacotherapy. Pharmacol Rev 58:389 462

Piomelli D (2003) The molecular logic of endocannabinoid signalling. Nat Rev Neurosci 4:873-884

Ramanathan L, Gulyani S, Nienhuis R, Siegel JM (2002) Sleep deprivation decreases superoxide dismutase activity in rat hippocampus and brainstem. Neuroreport 13:1387-1390

Schreiber D, Harlfinger S, Nolden BM, Gerth CW, Jaehde U, Schomig E, Klosterkotter J, Giuffrida A, Astarita G, Piomelli D, Markus Leweke F (2007) Determination of anandamide and other fatty acyl ethanolamides in human serum by electrospray tandem mass spectrometry. Anal Biochem 361:162-168 